\section{IN BRIEF}

\section{VASCULITIS}

\section{IVIG and ciclosporin for Kawasaki disease}

Intravenous immunoglobulin (IVIG) plus ciclosporin reduced the incidence of coronary artery abnormalities compared with IVIG alone $(95 \% \mathrm{Cl} 0.25-0.86 ; P=0.010)$ in a phase III study of 173 children with Kawasaki disease who were predicted to be unlikely to respond to IVIG therapy. Responsiveness to IVIG therapy was predicted on the basis of a composite risk score and individuals with pre-existing coronary artery abnormalities were excluded from the study. IVIG plus ciclosporin was safe and effective at 12 weeks, and no differences in adverse events were reported between treatment groups.

ORIGINAL ARTICLE Hamada, H. et al. Efficacy of primary treatment with immunoglobulin plus ciclosporin for prevention of coronary artery abnormalities in patients with Kawasak disease predicted to be at increased risk of non-response to intravenous immunoglobulin (KAICA): a randomised controlled, open-label, blinded-endpoints, phase 3 trial. The Lancet 393, 1128-1137 (2019)

\section{PAEDIATRIC RHEUMATOLOGY}

\section{Recombinant IL-1 Ra as first-line therapy in SJIA}

A treat-to-target approach using recombinant IL-1 receptor antagonist (rlL-1Ra; anakinra) as a first-line monotherapy in newly diagnosed patients with systemic juvenile idiopathic arthritis (sJIA; $n=42$ ) resulted in inactive disease in $95 \%$ of patients and inactive disease (off medication) in $73 \%$ of patients at 5 years follow-up. The treat-to-target strategy involved rapidly stepping up the dose of $\mathrm{rlL}-1 \mathrm{Ra}$ from $2 \mathrm{mg} / \mathrm{kg} /$ day to $4 \mathrm{mg} / \mathrm{kg} /$ day if fever persisted 3 days after starting therapy, followed by the addition of glucocorticoids and/or switching to an alternative therapy if disease persisted.

ORIGINAL ARTICLE ter Haar, N. M. et al. Treat-to-target using first-line recombinant interleukin-1 receptor antagonist monotherapy in new-onset systemic juvenile idiopathic arthritis: results from a five year follow-up study. Arthritis Rheumatol. https://doi.org/ 10.1002/art.40865 (2019)

\section{SPONDYLOARTHRITIS}

\section{New species of gut bacteria associated with AS}

A genomic screen of uncultivated human gut microorganisms from diverse global locations has revealed 2,058 previously unknown operational taxonomic units (OTUs) at a species level. One of these newly identified gut bacteria, OTU-14148, a species in the Negativicutes class, was associated with ankylosing spondylitis (AS). OTU-14148 was present at lower amounts in the stool of patients with AS than in that of healthy individuals and had an eightfold greater association with AS than the next most strongly associated species.

ORIGINAL ARTICLE Nayfach, S. et al. Novel insights from uncultivated genomes of the global human gut microbiome. Nature https://doi.org/10.1038/s41586-019-1058-x (2019)

\section{$\Rightarrow$ MYOSITIS}

\section{Serum biomarkers of disease activity in JDM}

Galectin 9 and CXC-chemokine ligand 10 (CXCL10) have been validated as serum biomarkers of disease activity in a group of 125 patients with juvenile dermatomyositis (JDM) from three international cohorts. The combination of high concentrations of galectin 9 and CXCL10 was better able to differentiate patients with JDM with active disease from those in remission (area under curve (AUC) 0.86-0.90) than the standard marker creatine kinase (AUC 0.66-0.68), and could also predict flare several months before onset. Galectin 9 and CXCL10 were also predictive of active disease in adults with dermatomyositis.

ORIGINAL ARTICLE Wienke, J. et al. Galectin- 9 and CXCL10 as biomarkers for disease activity in juvenile dermatomyositis: a longitudinal cohort study and multi-cohort validation. Arthritis Rheumatol. https://doi.org/10.1002/art.40881 (2019)

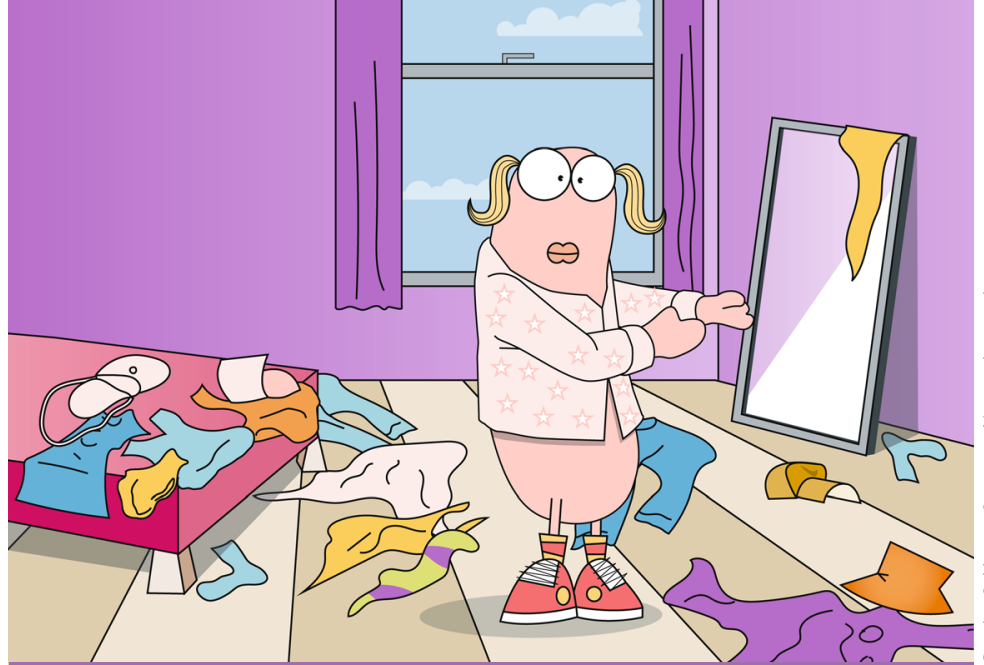

$\Rightarrow$ AUTOIMMUNITY

\title{
Bacterial cell wall offcasts linked to autoimmune disease
}

Gut microorganisms continuously shed peptidoglycans from their cell walls as they grow and divide, and these offcast molecules are capable of crossing the gut barrier and entering the circulation. According to new research published in Nature Microbiology, the neutralization of circulating peptidoglycans was sufficient to halt disease in several mouse models of autoimmunity, suggesting that peptidoglycans could be a new therapeutic target.

"The human microbiota affects many aspects of host physiology and has been associated with numerous diseases; however, how the microbiota generates systemic effects and affects extra-intestinal organs remains unclear," explains corresponding author Yue Wang.

Having previously shown that bacterial peptidoglycans are present in the serum of healthy individuals, Wang and colleagues developed an anti-muramyl-L-alanineD-isoglutamine (MDP) antibody called 2E7. This antibody enabled them to quantify and neutralize serum peptidoglycan by targeting MDP, a conserved structure within peptidoglycan. Using 2E7, the researchers confirmed the presence of MDP in the blood of all but 4 of the 340 healthy individuals tested, albeit at varying concentrations. Interestingly, serum concentrations of MDP were higher in patients with systemic lupus erythematosus $(n=190)$ or rheumatoid arthritis $(n=84)$ than in the healthy individuals, suggesting a link to autoimmune disease.
"We used two mouse models of autoimmune arthritis, collageninduced arthritis (CIA) and collagen antibody-induced arthritis (CAIA), to see how the manipulation of circulating peptidoglycan would affect the development of disease," says Wang.

Increasing the concentration of MDP in mice using an osmotic pump exacerbated CIA compared with giving saline, whereas administering 2E7 to reduce the concentration of MDP in mice reduced CIA severity compared with giving a control antibody. In the CAIA model, administration of 2E7 before the induction of disease almost completely prevented the development of arthritis.

Previous studies have determined that MDP is recognized by the pattern recognition receptor NOD2, which triggers pro-inflammatory signalling pathways. Accordingly, 2E7 was unable to prevent CAIA in mice that lacked NOD2, suggesting that neutralization of MDP by $2 \mathrm{E} 7$ ameliorated arthritis by reducing NOD2 signalling.

"Moving forward, we want to modify 2E7 for use in humans," says Wang. "We plan to carry out large-scale clinical studies to establish correlations between circulating peptidoglycan and immune-mediated diseases."

\section{Joanna Collison}

ORIGINAL ARTICLE Huang, Z. et al. Antibody neutralization of microbiota-derived circulating peptidoglycan dampens inflammation and ameliorates autoimmunity. Nat. Microbiol. https://doi.org/10.1038/s41564-019-0381-1 (2019) 